\title{
Atemwegskrankheiten in der Landwirtschaft
}

Landwirte haben im Vergleich zu anderen Berufsgruppen ein besonders hohes Allergisierungsrisiko. Chemisch-irritative Substanzen in den Futter- und Düngemitteln aber auch Getreidestäube sind häufige Allergieauslöser. Auf einem Symposium in Freiburg wurden nicht nur die Ursachen von Atemwegserkrankungen bei Landwirten diskutiert, es ging auch darum, wie man den Betroffenen, deren Lebensqualität oft stark beeinträchtigt ist, helfen kann.

llergien werden in der Landwirtschaft häufig durch Futtermittel und die Bedingungen, die eine sog. „optimale“ Zucht vorgibt, begünstigt. Hier sind insbesondere die Schweinezüchter betroffen, erläuterte D. Nowak, Hamburg. Mastschweineund Ferkelhalter haben mit bis $\mathrm{zu}$ $40 \%$ relativ hohe Prävalenzen für Atemwegssymptome. Je mehr Schweine ein Landwirt besitzt, um so höher scheint sein Allergierisiko. Die Schweine selbst induzieren dagegen höchst selten eine Typ-I-Allergie.

Allergenes Potential steckt nicht nur in Futter-, sondern auch in Düngemitteln. Häufig und zugleich als Allergen oft übersehen ist Rizinus, ein beliebtes Düngemittel. Einen ähnlich hohen Sensibilisierungsindex hat Fischmehl (vgl. Kasten).

\section{Cave Milben!}

Als starke Allergenträger gehören die Vorratsmilben zu den häufigsten respiratorischen Allergieauslösern in der Landwirtschaft, betonte R. Kroidl, Stade. Vorratsmilben kommen vor allem im Stall und im Futter vor. Von 3000 Landwirten, die wegen pulmonaler Probleme einen Lungenfacharzt aufsuchten, waren $25 \%$ auf Hausstaubmilben und $12,5 \%$ auf Vorrats- milben sensibilisiert. In einer Untersuchung mit 42 Landwirten und 48 Nicht-Landwirten war die Hälfte der Landwirte auf Vorratsmilben sensibilisiert. Im Vergleich zu den NichtLandwirten zeigten die Landwirte wesentlich häufiger einen positiven bronchialen Provokationstest auf Vorratsmilben.

\section{Welche Schutzmaßnahmen sind sinnvoll?}

Sensibilisierte Landwirte sollten einige Regeln in den Stallungen beachten: Natürliche Lüftungen sind wenig effizient und daher allergiefördernd. Ungünstig sind überdies mechanische Fütterungen und der häufige Einsatz von Desinfektionsmitteln. Zur Einstreuung in den Ställen sollten keine Sägespäne verwendet werden. Grundsätzlich ziehen Tätigkeiten, bei denen Staub aufgewirbelt wird, wie etwa das Ausmisten des Stalles per Hand, eine hohe Prävalenz für arbeitsplatzbezogene Atemwegserkrankungen nach sich.

Als Schutzmaßnahmen stehen dem Landwirt neben der Karenz nur wenige Möglichkeiten zur Verfügung. Der Wert von Atemschutzmasken ist umstritten; untauglich sind auf jeden Fall grobe Staubmasken. Neuere Atem-

\section{Sensibilisierungs- Index}

Der Sensibilisierungsindex ist der Prozentsatz sensibilisierter Personen aus der Gesamtzahl exponierter Personen in einer Berufsgruppe. Er beträgt für folgende, in der Landwirtschaft häufige Allergene:

- Futtermittelstäube: bis $45 \%$

- Rizinusschrot und -dünger: $41 \%$ bis $50 \%$

- Fischmehl: 50\%

- Kraftfutter (Soja, Tapioka, Luzerne): $10 \%$

- Getreidestäube: $18 \%$

schutzhelme führen die allergenen Partikel in einen separaten Behälter ähnlich einem Staubsauger - ab.

Schweinezüchtern empfiehlt A. Knoblauch, St. Gallen, Flüssigfutter oder pelliertes Futter zu verwenden. Das tägliche Abspritzen der Tiere mit Wasser oder auch Öl kann zumindest die Symptomatik lindern. Grundsätzlich sollten Wohnhaus und Stallungen getrennt sein.

\section{Schwierigkeiten der Diagnostik und der Begutachtung}

Entschädigungsleistungen wegen berufsbedingter Atemwegserkrankungen erhalten Landwirte nur selten. Problematisch ist bereits der Nachweis der Berufsspezifität. Darüber hinaus entwickeln viele Landwirte trotz verschlechterter FEV1-Werte keine manifeste obstruktive Atemwegserkrankung.

Dosis-Wirkungs-Beziehungen sind selten nachzuweisen, am ehesten noch für Endotoxin: Pro $100 \mathrm{ng} / \mathrm{ml}$ Endotoxin-Aufnahme nimmt der FEV1-Wert immerhin um gut $200 \mathrm{ml}$ ab. Ähnliches gilt aber beispielsweise nicht für Ammoniak. Hier treten Symptome in der Regel nur bei langandauernder Exposition auf, häufig sogar nur in Verbindung mit einer anderen Noxe.

(bp)

Symposium „Obstruktive Atemwegskrankheiten in der Landwirtschaft" anläßlich des DGP, Freiburg, 6.3.1997. 